\title{
Functional protease profiling with reporter peptides in serum specimens of colorectal cancer patients: demonstration of its routine diagnostic applicability
}

Peter Findeisen ${ }^{1 *}$, Victor Costina ${ }^{1}$, Diego Yepes ${ }^{1}$, Ralf Hofheinz ${ }^{2}$ and Michael Neumaier ${ }^{1}$

\begin{abstract}
Background: The progression of many solid tumors is characterized by the release of tumor-associated proteases and the detection of tumor specific proteolytic activity in serum specimens is a promising diagnostic tool in oncology. Here we describe a mass spectrometry-based functional proteomic profiling approach that tracks the ex-vivo degradation of a synthetic endoprotease substrate in serum specimens of colorectal tumor patients.

Methods: A reporter peptide (RP) with the amino acid sequence WKPYDAAD was synthesized that has a known cleavage site for the cysteine-endopeptidase cancer procoagulant (EC 3.4.22.26). The RP was added to serum specimens from colorectal cancer patients $(n=30)$, inflammatory controls $(n=30)$ and healthy controls $(n=30)$ and incubated under strictly standardized conditions. The proteolytic fragment of the RP was quantified with liquid chromatography / mass spectrometry (LC/MS).
\end{abstract}

Results: RP-spiking showed good intra- and inter-day reproducibility with coefficients of variation (CVs) that did not exceed a value of $10 \%$. The calibration curve for the anchor peptide was linear in the concentration range of $0.4-50 \mu \mathrm{mol} / \mathrm{L}$. The median concentration of the RP-fragment in serum specimens from tumor patients (TU: $17.6 \mu \mathrm{mol} / \mathrm{L}, \mathrm{SD} 9.0$ ) was significantly higher when compared to non-malignant inflammatory controls (IC: $11.1 \mu \mathrm{mol} / \mathrm{L}, \mathrm{SD}$ 6.1) and healthy controls (HC: $10.3 \mu \mathrm{mol} / \mathrm{L}, \mathrm{SD} 3.1$ ). Highest area under receiver operating characteristic (AUROC) values were seen for discrimination of TU versus HC (0.89) followed by TU versus IC (0.77). IC and $\mathrm{HC}$ could barely be separated indicated by an AUROC value of 0.57 . The proteolytic activity towards the RP was conserved in serum specimens that were kept at room temperature for up to 24 hours prior to the analysis.

Conclusion: The proteolytic cleavage of reporter peptides is a surrogate marker for tumor associated proteolytic activity in serum specimens of cancer patients. A simple, robust and highly reproducible LC/MS method has been developed that allows the quantification of proteolytic fragments in serum specimens. The preanalytical impact of sample handling is minimal as the tumor-associated proteolytic activity towards the reporter peptide is stable for at least up to $24 \mathrm{~h}$. Taken together, the functional protease profiling shows characteristics that are in line with routinely performed diagnostic assays. Further work will focus on the identification of additional reporter peptides for the construction of a multiplex assay to increase diagnostic accuracy of the functional protease profiling.

Keywords: Functional protease profiling, Serum, Colorectal cancer, Cancer procoagulant diagnosis, Reporter peptide, Mass spectrometry

\footnotetext{
* Correspondence: peter.findeisen@umm.de

'Institute for Clinical Chemistry, Medical Faculty Mannheim of the University of Heidelberg, University Hospital Mannheim, Theodor-Kutzer-Ufer 1-3, Mannheim 68167, Germany

Full list of author information is available at the end of the article
} 


\section{Background}

Proteases play an important role in different biological processes including cell differentiation, inflammation and tissue remodelling, haemostasis, immunity, angiogenesis, apoptosis and malignant disease [1]. Specifically, proteases are well known factors to promote local progression and distant metastasis of colorectal cancer and many other solid tumors [2,3]. Furthermore, there is increasing evidence that proteases also have key functions in early stages of tumor development [4]. The tumorassociated proteases are either secreted directly by the tumor or originate from surrounding connective tissue and infiltrating leucocytes as a result of tumor-stroma interaction [5]. Some tumor-associated proteases like cathepsins, matrix-metalloproteases, kallikreins and cancer procoagulant $(\mathrm{CP})$ are released into the bloodstream and can be used for diagnostic and prognostic purposes [6-10]. Tumor-associated protease activity in serum specimens of cancer patients can be monitored using synthetic substrates that are selectively cleaved by the protease of interest [6-9]. With the use of appropriate synthetic reporter-peptides (RPs) for spiking of serum specimens, the reaction conditions that comprise substrate concentration, incubation time and buffer composition can be optimized and standardized accordingly [11]. Furthermore, the proteolytic fragments accumulate to the level that they become readily detectable by mass spectrometry [8]. This approach is similar to established diagnostic assays measuring the proteolytic activity of distinct enzymes, e.g., coagulation factors [12].

Recently, we have described a functional protease profiling approach using a reporter peptide that is cleaved by the tumor associated protease cancer procoagulant (EC 3.4.22.26) [8]. However, the analysis of proteolytic fragments was performed with MALDI-TOF mass spectrometry that is only a semi-quantitative method [13] with limited inter-day reproducibility [8]. Furthermore, proteolytic fragments had to be extracted from serum specimens with serial affinity purification that is a rather laborious method with limited throughput and reproducibility. To alleviate these restrictions, we have developed a robust and highly reproducible liquid chromatography-mass spectrometry (LC-MS) assay for the absolute quantification of a targeted proteolytic fragment.

Serum has a high intrinsic proteolytic activity that leads to continuous processing of proteins and peptides [14]. To protect the reporter peptide from unwanted and unspecific processing by exopeptidases the cleavage site WKPYDAAD is flanked by aminohexanoic acid (see Table 1). When the reporter peptide is cleaved by the endoprotease cancer procoagulant after the tyrosine $(\mathrm{Y})$ [15], the resulting free amino-terminus of the intermediate fragment is rapidly trimmed down by aminopeptidases [8]. This results in the accumulation of a protease resistant
Table 1 Peptide sequences of reporter peptide, anchor peptide and internal standard

\begin{tabular}{llcc}
\hline Name & Peptide sequence & $\begin{array}{c}{[\mathbf{M}+\mathbf{H}]^{2+}} \\
\text { observed }\end{array}$ & $\begin{array}{c}{[\mathbf{M}+\mathbf{H}]^{\mathbf{1 +}}} \\
\text { theoretical } \\
\text { (monoisotopic) }\end{array}$ \\
\hline CP-RP & $\begin{array}{l}\text { Ahx-WKPYDAAD- } \\
\text { Ahx-ateeqlkv }\end{array}$ & $2.090,06$ \\
CP-AP & Ahx-ateeqlkv & 515,795 & $1.030,59$ \\
IS & Ahx-ateevlkl & 508,300 & $1.015,61$ \\
\hline
\end{tabular}

CP-RP: Cancer Procoagulant-Reporter Peptide. CP-AP: Cancer ProcoagulantAnchor Peptide. IS: Internal Standard. Ahx: amino hexanoic acid. Lower case letters indicate D-amino acids.

anchorpeptide (CP-AP) that consists of aminohexanoic acid and D-aminoacids (see Table 1). The anchorpeptide was quantified by liquid chromatography / mass spectrometry (LC/MS) with good reproducibility that is in line with routinely performed diagnostic tests.

The sufficient preanalytical stability of biomarkers is a prerequisite for routine diagnostic use and we could demonstrate that the tumor-associated proteolytic activity towards the reporter peptide is preserved for up to $24 \mathrm{~h}$. Furthermore a small proof-of-concept experiment $(n=90)$ was performed to demonstrate the diagnostic power of functional protease profiling with reporter peptide spiking. Systemic inflammation has been recognized as serious threat for cancer biomarker discovery [16] and we selected the collective of control individuals accordingly. The concentrations of proteolytic fragments were significantly higher in serum specimens from tumor patients (TU) when compared to serum from inflammatory controls (IC) and healthy controls (HC). This indicates the presence of the tumor-associated protease cancer procoagulant that is associated with an increased cleavage of the reporter peptide in serum specimens of tumor patients.

Here we present a method to monitor controlled, $e x-$ vivo peptide breakdown in serum samples using LC/MS with absolute quantification of the respective fragment that might lead to an activity based approach for biomarker discovery and validation.

\section{Results}

LC-MS analysis and absolute quantification of the anchor peptide

The proteolytic cleavage of the reporter peptide (CP-RP) by the endoprotease cancer procoagulant results in an accumulation of the anchor peptide (CP-AP). The amino acid sequence WKPYDAAD of CP-RP is specifically cleaved after the aminoacid tyrosine $(\mathrm{Y})$ by the endoprotease cancer procoagulant prior to further processing by serum exopeptidases $[8,15]$. Finally, the proteaseresistant anchor peptide (CP-AP) m/z 515.795 which consists of the linker and D-amino acids (Table 1) is 
accumulating and high concentration is a surrogate marker for increased proteolytic activity of cancer procoagulant. Figure 1 gives a representative example of the LC/MS results for CP-AP. Figure $1 \mathrm{~A}$ shows the extracted ion chromatogram (XIC) of CP-AP and labelling of the respective peak area that was used for quantification. Figure $1 \mathrm{~B}$ shows the corresponding mass spectrum within the selected mass window ranging from $\mathrm{m} / \mathrm{z} 250$ to $\mathrm{m} / \mathrm{z} 600$. Note that only one peak with the respective isotopic pattern exceeded the signal intensity of $2 \times 10^{7}$ [a.u.]. This $\mathrm{m} / \mathrm{z} 515.795$ was expected to be the doubly charged molecule CP-AP (Table 1) and the sequence was verified by tandem mass spectrometry (Additional file 1: Figure S1). The mass spectra of the internal standard (IS) are of equal quality regarding the signal to noise ratio (data not shown). A calibration curve was prepared using pooled serum of healthy controls that was spiked with four different concentrations of CP-AP ranging from 0.4 to $50 \mu \mathrm{mol} / \mathrm{L}$. The linearity of the calibration curve within this concentration range was good with a coefficient of determination $\left(R^{2}\right)$ of 0.992 (Figure 2).

\section{Optimization of incubation time and reproducibility of RP-spiking}

The quantification of the anchor peptide CP-AP is performed as mass-spectrometric endpoint-assay and the appropriate incubation time has to be determined. As expected, the concentration of CP-AP is constantly increasing during prolongation of the incubation time from $3 \mathrm{~h}$ to $6 \mathrm{~h}$ and $22 \mathrm{~h}$ (Figure 3A). The accumulation of CP-AP is approximately five times faster in the tumor serum (QCT), when compared to a healthy control specimen $(\mathrm{QCH})$ as indicated by the linear regression graphs with slopes of 0.836 and 0.164 respectively (Figure 3A). The incubation for $22 \mathrm{~h}$ seems to be preferable as reproducibility of measurements is improved with increasing signal intensity that is associated with prolonged incubation time [17]. The CVs are inversely correlated to the signal intensity and range from $6.8 \%$ to $3.0 \%$ for CP-AP concentrations of $0.33 \mu \mathrm{mol} / \mathrm{L}$ and $18.7 \mu \mathrm{mol} / \mathrm{L}$ respectively (Figure $3 \mathrm{~B}$ ). Consequently, an incubation period of $22 \mathrm{~h}$ was chosen for any further experiments.

\section{Inhibition of proteolytic reaction with iodoacetamide}

The cysteine-endoprotease cancer procoagulant can specifically be inhibited by iodoacetamide [18] and different concentrations of protease inhibitor were added to spiked serum specimens of a tumor patient. As expected, the concentration of $\mathrm{CP}$-AP is inversely proportional to the amount of iodoacetamide concentrations of serum specimens that were spiked with CP-RP. After $22 \mathrm{~h}$ of incubation the amount of CP-AP that accumulated in the serum specimen was taken as $100 \%$. In the presence of 5,25 and $100 \mathrm{mmol} / \mathrm{L}$ jodoacetamide, the CP-AP concentration was reduced down to $88 \%, 63 \%$ and $25 \%$ respectively (Additional file 2: Figure S2).
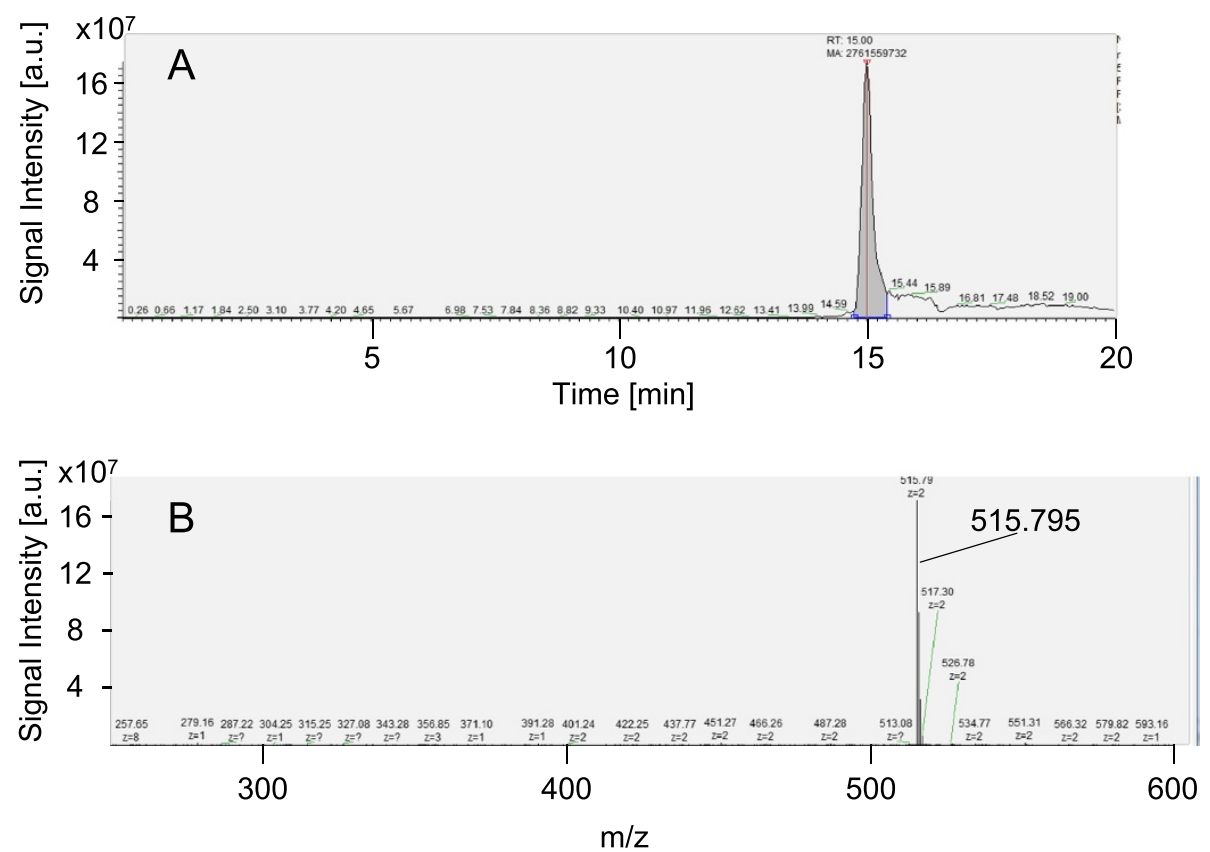

Figure 1 Exemplary LC/MS results. LC/MS results of the calibration standard with CP-AP concentration of $0.4 \mu$ mol/L (A) Extracted ion chromatogram (XIC) of CP-AP with extracted mass of $515.795+/-0.005$. The peak area of the respective $\mathrm{m} / \mathrm{z} 515.795$ is filled in grey and was used for quantification. (B) ESI mass spectrum of the anchor peptide eluting at $15+/-1$ min. 


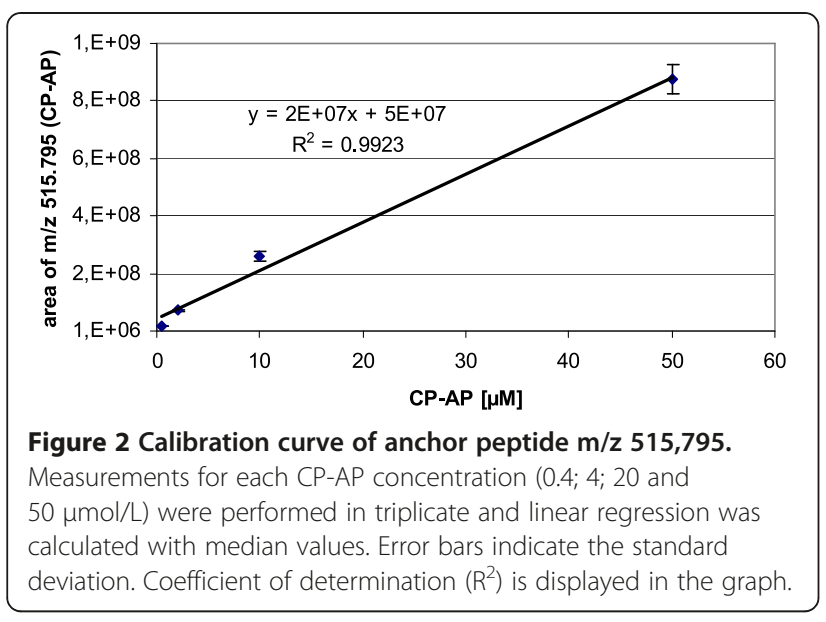

Preanalytical stability of cancer procoagulant activity

Serum specimens from 6 tumor patients were aliquoted and stored $0,3,6$ and $24 \mathrm{~h}$ at room temperature prior to freezing at $-80^{\circ} \mathrm{C}$. After thawing, reporter peptide CP$\mathrm{RP}$ was added to serum specimens and incubated $22 \mathrm{~h}$ under standardized conditions as described in materials and methods. The concentrations of CP-AP in the serum specimens without preanalytical time delay $(0 \mathrm{~h})$ ranged from $4.27 \mu \mathrm{mol} / \mathrm{L}$ to $13.14 \mu \mathrm{mol} / \mathrm{L}$ and were set to $100 \%$. Compared to freshly prepared specimens $(0 \mathrm{~h})$ the CP-AP concentrations after 3, 6 and 24 h of preanalytical time had median values of $103 \%, 102 \%$ and $97 \%$ respectively (Figure 4). The concentrations of CP-AP in serum specimens with prolonged preanalytical time span ( $3 \mathrm{~h}, 6 \mathrm{~h}, 24 \mathrm{~h}$ ) were not significantly different from concentrations that were measured in fresh specimens $(0 \mathrm{~h})$. This indicates that cancer procoagulant activity towards the reporter peptide is stable at least over a preanalytical time period of $24 \mathrm{~h}$.

\section{Functional protease profiling}

Finally, we set up a proof-of-concept experiment to elucidate the applicability of protease profiling for diagnostic purposes. However, the implementation of MS as a routine diagnostic tool clearly depends on good interday reproducibility of the method. Three aliquots of a serum specimen from one tumor patient were randomly integrated into small series of serum specimens from patients and control individuals on four consecutive days. The median concentration of CP-AP was $31.9 \mu \mathrm{mol} / \mathrm{L}$ with $\mathrm{SD}$ of $3.3 \mu \mathrm{mol} / \mathrm{L}$ and $\mathrm{CV}$ of $10.2 \%$ (Additional file 3: Figure S3). As expected, the inter-day reproducibility is not as good as the intra-day reproducibility (see Figure 3B). However, CVs of $10 \%$ or even
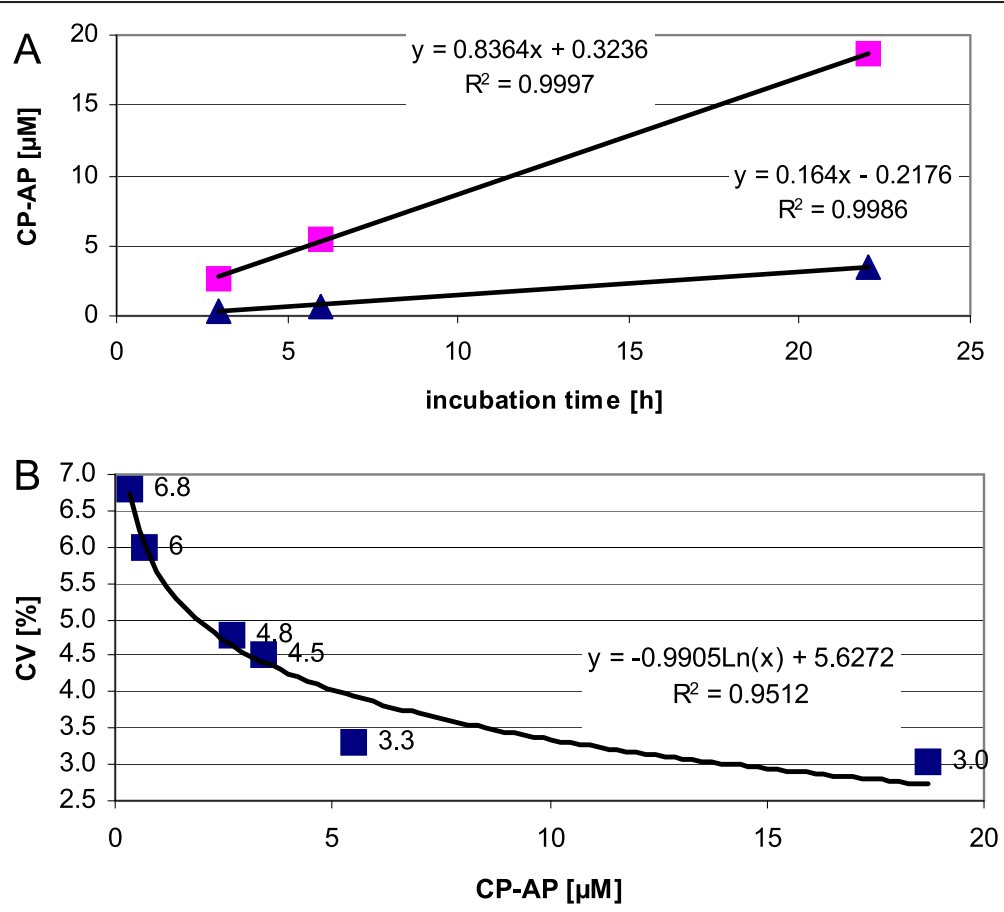

Figure 3 Kinetic measurements of CP-AP in pooled serum specimens of tumor patients and healthy controls. (A) Accumulation of CP-AP correlates with incubation time. Linear regression was calculated from median values of five measurements. Squares: pooled serum specimen from tumor patients. Triangles: pooled serum specimen from healthy controls. The equations of the linear regression and coefficients of determination $\left(R^{2}\right)$ are displayed in the graph. (B) Intraday-reproducibility. Inverse correlation of concentrations of CP-AP and coefficients of variations (CVs) for five repetitive measurements. The CVs (y-values) are shown next to the squares in the graph. A logarithmic regression has been calculated with Excel (Microsoft) and the equation and coefficients of determination $\left(R^{2}\right)$ are also displayed in the graph. 


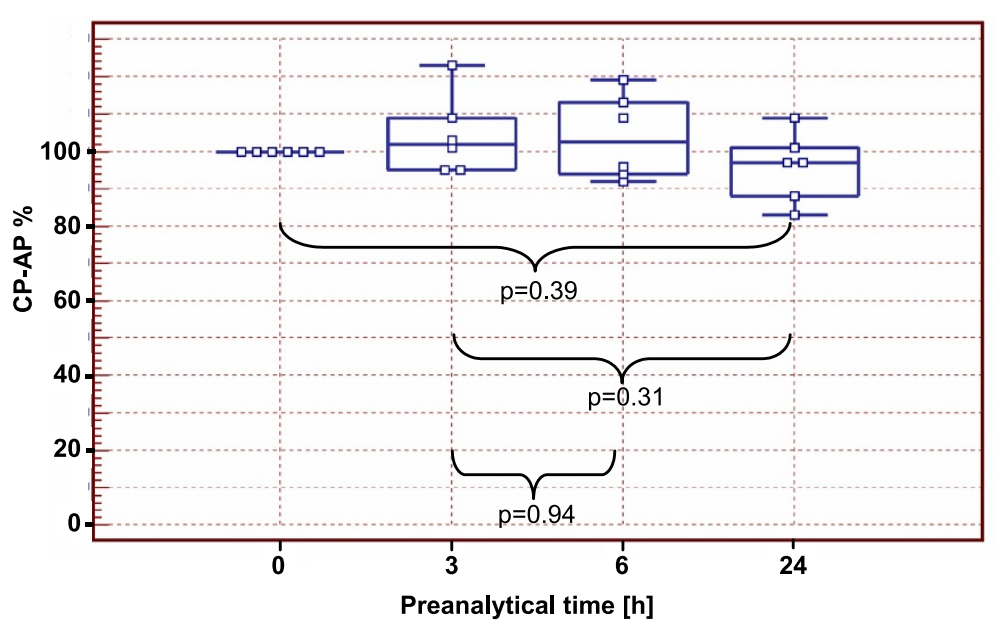

Figure 4 Preservation of protease activity in a preanalytical time period of $\mathbf{2 4}$ h. Aliquots of serum specimens from 6 tumor patients were frozen at $-80^{\circ} \mathrm{C}$ directly after centrifugation $(0 \mathrm{~h})$ or after prolonged preanalytical time span of $3 \mathrm{~h}, 6 \mathrm{~h}$, and $24 \mathrm{~h}$. After thawing, specimens were spiked with CP-RP and incubated for $22 \mathrm{~h}$ prior to peptide extraction with TCA and LC-MS. CP-AP peak areas were extracted from the data. The $\mathrm{CP}-\mathrm{AP}$ concentrations of the freshly obtained serum aliquots $(0 \mathrm{~h})$ were set to $100 \%$. In the box plot the central box represents the values from the lower to upper quartile ( 25 to 75 percentile). The middle line represents the median. The horizontal line extends from the minimum to the maximum value. P-values of the Mann-Whitney test are indicated.

more are acceptable for many routine laboratory assays [19].

Serum specimens from patients with metastatic colorectal tumors $(\mathrm{TP}=30)$, patients without malignant disease but elevated acute phase protein CRP $(\mathrm{IC}=30)$ and healthy controls $(\mathrm{HC}=30)$ were spiked with $\mathrm{CP}-\mathrm{RP}$ and internal standard (IS). Samples were incubation for $22 \mathrm{~h}$ and sample preparation prior to LC-MS was performed as described in materials and methods. The median concentrations of CP-AP in the collectives of healthy controls (HC), inflammatory controls (IC) and tumor patients (TP) were 10.3 (SD 3.1), 11.1 (SD 6.1) and 17.6 (SD 9.0) respectively (Figure 5A). The D'AgostinoPearson test was used to asses the normal distribution within the reporter peptide concentrations. For HC and IC the p-values were higher than 0.05 indicating a normal distribution. However, for TU the p-value was $<0.05$ and the hypothesis that the distribution of the observations in the sample is normal, was rejected. Accordingly, further data analysis was performed with the nonparametric Mann-Whitney test. The concentrations of CP-AP were not significantly different, when $\mathrm{HC}$ versus IC was compared with the Mann-Whitney test $(p=0.337)$. In contrast, the comparison of $\mathrm{HC}$ versus TP and IC versus TP showed statistically significant differences with $\mathrm{p}$ values below 0.005 (Figure 5A). The diagnostic accuracy for discrimination of healthy controls and tumor patients was calculated with receiver operating characteristics (ROC) that had an area under the curve (AUC) of 0.89. The ROC-AUC for discrimination of inflammatory controls and tumor patients had a value of 0.77 . The $95 \%$ confidence intervals ranged from 0.787 to 0.958 and from 0.646 to 0.871 respectively. In contrast, inflammatory controls and healthy controls could not be differentiated with a ROC-AUC of 0.57 with $95 \%$ confidence interval ranging from 0,438 to 0,699 (Figure 5B). These data suggest that the activity of the tumor-associated endoprotease cancer procoagulant is increased in serum specimens of tumor patients when compared to healthy and inflammatory controls.

\section{Discussion}

The dysregulation of protease activity plays an important role for the initiation and progression of malignant disease $[1,4]$. Tumor-associated proteases like matrix metalloproteases, cathepsins, kallikrein related peptidases and members of the plasminogen activator system are secreted into the bloodstream and might be candidates for functional protease profiling (for review see [20]). Specifically, the tumor-associated protease cancer procoagulant is secreted from numerous malignancies including colorectal cancer into the bloodstream [21]. Under in vivo conditions this can cause paraneoplastic coagulopathy throughout cleavage and activation of the coagulation factor X heavy chain (P00742) [22]. The reporter peptide CP-RP comprises the cleavage site WKPYDAAD that is part of the coagulation factor $\mathrm{X}$ and is preferably cleaved in serum specimens of tumor patients [8]. Adding reporter peptides to serum specimens enables the monitoring of tumor-related proteolytic activity for diagnostic use [7-9,23,24]. Furthermore, reporter peptide spiking offers major advantages over native MS-based peptide profiling concerning the standardization of preanalytical variabilities $[6,11]$. The 


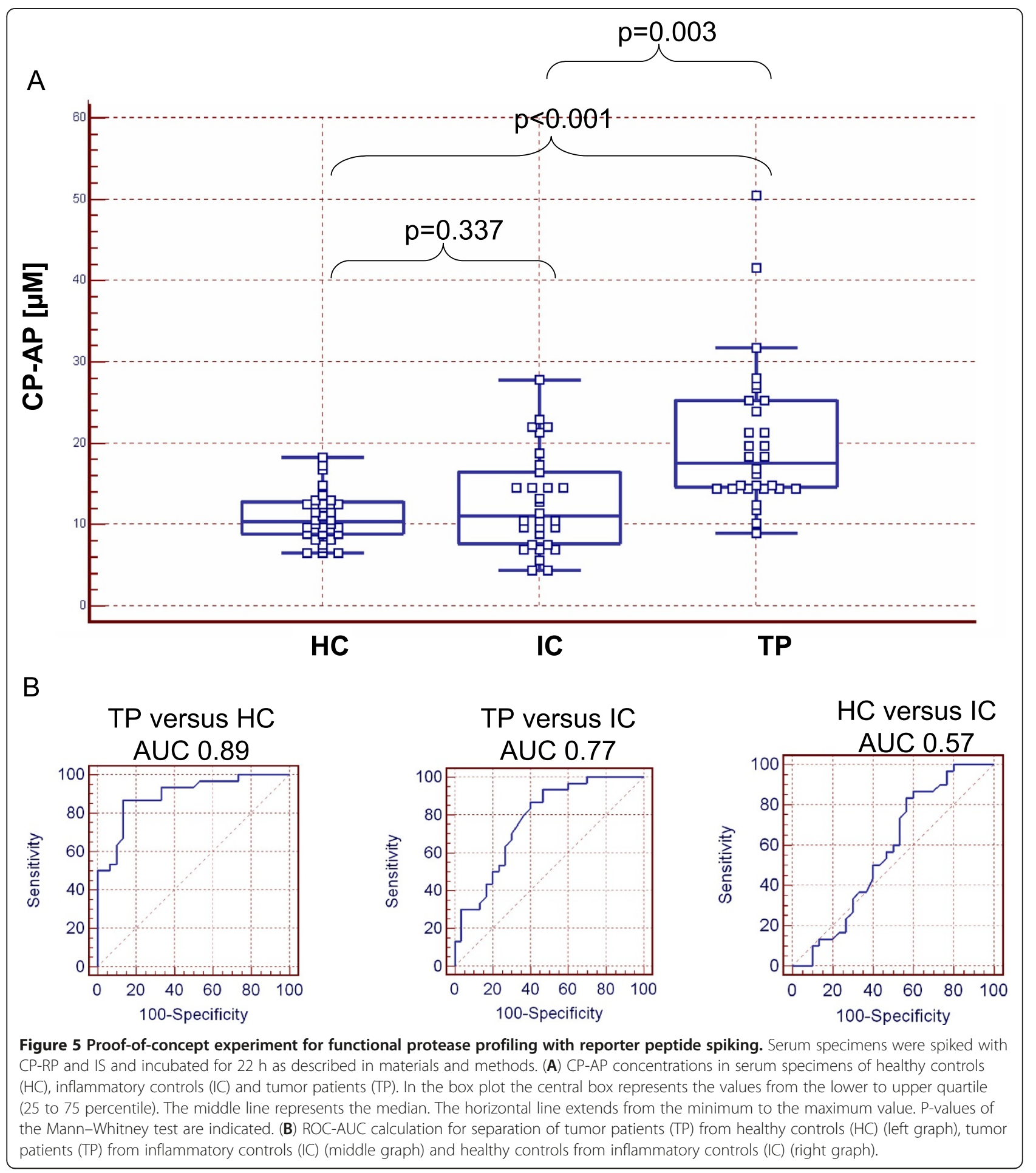

main focus of our present work was to optimize functional protease profiling with respect to simplified sample preparation and increased inter-day reproducibility to make it amenable as a laboratory assay for routine diagnostic use.

Recently, a sample clean-up with trichloroacetic acid (TCA) has been described that showed a sufficient recovery for peptides with a molecular weight of less than $3000 \mathrm{Da}$ [25]. Furthermore, the LC-MS technique is the method of choice for the reproducible quantification of small molecules like peptides in clinical specimens [26], and accordingly this technology was selected for assay development. Even at low CP-AP concentrations of $0.4 \mu \mathrm{mol} / \mathrm{L}$ the extracted ion chromatogram of 
CP-AP with $\mathrm{m} / \mathrm{z} 515.795$ shows only one single peak (see Figure 1) and this excellent signal to noise ratio makes quantitative LC/MS analyses amenable [27,28]. Recently, criticism has been raised against functional protease profiling and it has been suggested to characterize the proteolytic activity in more detail [29]. Here, we demonstrate that the proteolytic processing of CP-RP and thus the accumulation of CP-AP can be inhibited by the addition of a protease inhibitor. Iodoacetamide is a known cysteine protease inhibitor and reacts readily with the free thiol of cysteine residues required for the hydrolyzing proteases such as cancer procoagulant $[18,30]$. The amount of CP-AP that is generated in the serum of cancer patients is inversely proportional to the concentration of iodoacetamide added (Additional file 2: Figure S2). This demonstrates that the cleavage of CP-RP and the accumulation of CP-AP is a specific reaction that is related to cysteinprotease activity.

Most interestingly, the proteolytic activity of serum specimens towards CP-RP is conserved for up to $24 \mathrm{~h}$ indicating a good preanalytical stability making it useful for diagnostic application (Figure 4).

One major challenge of functional protease profiling is the appropriate selection of exogenous reporter peptides, which are exclusively cleaved by tumor-associated proteases. However, serum is a difficult matrix with high intrinsic proteolytic activity caused by different endoproteases e.g. from the coagulation cascade and the complement system $[14,31,32]$ as well as a multitude of exoproteases [33]. Furthermore, the proteolytic profile in blood specimens is not only altered in malignant disease but also under non-malignant conditions e.g. inflammation [16]. In order to be useful for diagnostics, such proteolytic patterns must be distinguishable from e.g. the inflammatory responses in unrelated non-malignant conditions. As these patterns overlap to a great extent, the classification of tumour patients on the basis of proteolytic activity is a demanding task. Our study addresses this important question by demonstrating the diagnostic accuracy of functional protease profiling with exogenous reporter peptides in a proof-of-concept experiment including patients with inflammatory conditions during non-malignant diseases into the control cohort. Most importantly, there were no statistically significant differences of CP-AP concentrations between the healthy controls and inflammatory controls, while CP-AP concentrations were significantly higher in serum specimens from tumor patients (see Figure 5A). This indicates that changes of the proteolytic profile related to inflammation do not affect the specific processing of the reporter peptide CP-RP. However, we emphasize that this small proof-of-principle profiling experiment has serious shortcomings concerning the limited number of analyzed specimens and the selection of late-stage tumor patients with highly elevated CEA concentrations (see Table 2). Further studies will have to integrate also early tumor stages and in addition should evaluate the impact of therapeutic interventions to clarify the potential benefit of functional protease profiling. Finally, it is likely that tumor heterogeneity during progression of malignant disease may result in different protease patterns [34]. Based on the use of one single reporter peptide as shown here this needs to be accommodated to ensure high diagnostic accuracy and accordingly the areas under the ROC curves were 'only' 0.89 and 0.77 for the discrimination of tumor patients versus healthy controls and tumor patients versus inflammatory controls respectively (see Figure 5B). To increase the diagnostic accuracy of functional protease profiling, it seems reasonable to combine different reporter peptides for multiplex analysis that has potentially superior diagnostic accuracy [35]. To achieve this goal, it will be necessary to systematically identify reporter peptide sequences that are most efficiently cleaved by disease-specific proteases. However, any multiplex assay for functional protease profiling might implement the development of kinetic measurements and the need for chromogenic protease substrates

Table 2 Patient demographics and clinical characteristics

\begin{tabular}{|c|c|c|c|c|c|c|c|c|c|c|}
\hline \multirow[b]{2}{*}{ Classification } & \multicolumn{2}{|c|}{ Diagnosis } & \multicolumn{2}{|c|}{ CEA $[\mu \mathrm{g} / \mathrm{l}]$} & \multicolumn{2}{|c|}{ CRP [mg/l] } & \multicolumn{2}{|c|}{ Sex } & \multicolumn{2}{|c|}{ Age } \\
\hline & Disease & $\mathbf{n}$ & Mean & SD & Mean & SD & Male & Female & Mean & SD \\
\hline$\overline{\mathrm{HC}}$ & not reported & 30 & 3,3 & 1,3 & 3,3 & 2 & 10 & 20 & 50,0 & 9,4 \\
\hline \multirow[t]{6}{*}{ IC } & tissue damage & 13 & 2,8 & 1,4 & 146,9 & 61 & 19 & 11 & 68,9 & 12,2 \\
\hline & pneumonia & 7 & & & & & & & & \\
\hline & UTI & 4 & & & & & & & & \\
\hline & $\mathrm{IBD}$ & 2 & & & & & & & & \\
\hline & pancreatitis & 2 & & & & & & & & \\
\hline & sepsis & 2 & & & & & & & & \\
\hline TU & CRC & 30 & 597,6 & 1014,7 & 10,9 & 7 & 14 & 16 & 66,2 & 10,4 \\
\hline
\end{tabular}

HC; healthy controls. IC; inflammatory controls. TU; tumor patients. UTI; urinary tract infection. IBD; inflammatory bowel disease. Reference range of CEA: $<5 \mu g / l$. Reference range of CRP: $<5 \mathrm{mg} / \mathrm{l}$. 
[36]. Further work will focus on the identification of additional reporter peptides that are cleaved by other tumor-associated proteases e.g. metalloproteases, cathepsins or kallikreins in order to construct a multiplex protease profiling assay with increased diagnostic sensitivity and specificity.

\section{Conclusion}

Here we present an optimized LC/MS assay for the quantification of a reporter peptide fragment that correlates with tumor-associated proteolytic activity in serum specimens of colorectal cancer patients. With this improved method three major observations could be made: First, the reproducibility of the assay is excellent with coefficients of variation that did not exceed $10 \%$. Second, the tumor-associated proteolytic activity towards the reporter peptide is stable in serum specimens for up to 24 hours. Specifically, good reproducibility and sufficient preanalytical stability are major prerequisites of laboratory diagnostic assays. Third, inflammatory controls (IC) could fairly be separated from tumorpatients (TP) and this is most important as inflammation is an inherent component of cancer and many studies have identified biomarkers that are associated with inflammation rather than malignancy [16]. However, there is a considerable overlap concerning the concentration of CP-AP in serum specimens from controls and tumorpatients. The combination of multiple reporter peptides that are processed by different tumor-associated proteases will be necessary to increase diagnostic accuracy of functional protease profiling. However, if suitable reporter peptides are available, the simultaneous quantification of multiple anchorpeptides could easily be adopted for the presented LC/MS method.

\section{Materials and methods \\ Materials and chemicals}

The reporter peptide (CP-RP), the anchor peptide (CP$\mathrm{AP}$ ) and the internal standard (IS) (Table 1) were synthesized in the functional genome analysis laboratory of the German Cancer Research Centre (Heidelberg, Germany). HPLC-grade acetonitrile was purchased from Fisher Chemicals (Germany). Formic acid was purchased from Sigma (Germany). Phosphate buffered saline $\mathrm{pH} 7.4$ (PBS) was purchased from PAA Laboratories. Protease buffer: $200 \mathrm{~mol} / \mathrm{L}$ Tris $\mathrm{HCl}, 20 \mathrm{mmol} / \mathrm{L} \mathrm{CaCl}_{2}$, pH 7.8. Iodoacetamide and trichloroacetic acid were purchased from Sigma and Fluka respectively. All reagents and chemicals were at least of analytical grade.

\section{Serum samples}

Whole blood specimens were acquired from patients with metastatic colorectal tumors $(n=30)$ and patients without malignant disease but elevated acute phase protein CRP $(\mathrm{n}=30)$ at the University Hospital Mannheim. Blood from healthy control individuals $(n=30)$ was taken from employees of the University Hospital Mannheim during routine laboratory testing at the works doctor's office. Patient characteristics are summarized in Table 2. Blood collection was performed after we obtained institutional review board approval and patients' written informed consent. After a 30 min clotting time at room temperature the specimens were centrifuged at $20^{\circ} \mathrm{C}$ for $10 \mathrm{~min}$ at $3000 \mathrm{x} \mathrm{g}$. The serum was aliquoted and stored at $-80^{\circ} \mathrm{C}$ until further use. All serum specimens were refrigerated within 6 hours after blood withdrawal. Any handling and processing of serum specimens from tumor patients and controls was performed in a strictly randomized and blinded manner. Measurements of C-reactive protein (CRP) and carcinoembryonic antigene (CEA) were performed on the Dimension Vista ${ }^{\mathrm{TM}}$ System (Siemens).

\section{Sample preparation}

Serum specimens were diluted in the ratio of 1:3 with PBS to a final volume of $100 \mu \mathrm{L}$. The reporter peptide (CP-RP) and the internal standard (IS) were dissolved in protease buffer to a concentration of $100 \mu \mathrm{mol} / \mathrm{L}$ for $\mathrm{CP}-\mathrm{RP}$ and $20 \mu \mathrm{mol} / \mathrm{L}$ for the IS. The diluted serum (50 $\mu \mathrm{L})$ and the mix of RP and IS $(50 \mu \mathrm{L})$ were incubated at $37^{\circ} \mathrm{C}$ for $3 \mathrm{~h}, 6 \mathrm{~h}$ or $22 \mathrm{~h}$ as depicted in results. The incubation was terminated by adding $100 \mu \mathrm{L}$ of $10 \%$ (v/v) trichloroacetic acid (TCA) and the resulting mixture was kept at $4^{\circ} \mathrm{C}$ for $30 \mathrm{~min}$ prior to centrifugation for $15 \mathrm{~min}$. at $4^{\circ} \mathrm{C}$ and $12.000 \mathrm{rpm}$ in a microcentrifuge (Eppendorf). The supernatant was again centrifuged for $5 \mathrm{~min}$. at $4^{\circ} \mathrm{C}$ and $12.000 \mathrm{rpm}$ and $2 \mu \mathrm{L}$ of the supernatant were injected onto the HPLC-column.

\section{Liquid chromatography - mass spectrometry (LC-MS) analysis}

LC-MS was performed using a nano HPLC system (UltiMate3000, Dionex) coupled to a linear ion trap Fourier Transform Ion Cyclotron Resonance mass spectrometer (LTQ-FTICR, Thermo Fisher Scientific) with a chip interface (TriVersa NanoMate, Advion). Analytical chromatography of CP-AP and IS (see Table 1) was performed on a $75 \mu \mathrm{m}$ ID C-18 column (Dionex) with a flow of $300 \mathrm{~nL} / \mathrm{min}$ and a gradient from $20-35 \%$ of buffer B in 23 min.. The composition of buffer A was water with $0.1 \%$ formic acid and buffer $B$ was $80 \%$ acetonitrile with $0,08 \%$ formic acid. Each LC run was preceded by a blank run ensuring lack of carryover of the material from the previous run. MS analysis was performed in positive ion mode, with a mass range of $250-600 \mathrm{~m} / \mathrm{z}$. MS/MS analyses were performed on the reporter peptide fragment CP-AP for sequence confirmation. 


\section{Reproducibility of reporter peptide spiking}

To monitor the reproducibility of reporter peptide spiking, two distinct quality control samples were generated comprising serum specimens from five colorectal tumor patients (QCT) and five healthy control individuals $(\mathrm{QCH})$, respectively. Both samples were aliquoted and stored at $-80^{\circ} \mathrm{C}$ until further use. The QCT and QCHsamples were spiked with the reporter peptide and internal standard and incubated for $3 \mathrm{~h}, 6 \mathrm{~h}$ and $22 \mathrm{~h}$ at $37^{\circ} \mathrm{C}$ as described above. The proteolytic processing of the reporter peptide CP-RP resulted in the accumulation of CP-AP and the respective peak areas were used for quantification using LCQuan that is part of the Xcalibur software package (Thermo Fisher Scientific). Each QCspecimen was processed 5 times and median, standard deviation (SD) and coefficient of variation $(\mathrm{CV})$ of the m/z 515.795 peak was calculated with Microsoft Excel software.

\section{Statistics}

The D'Agostino-Pearson test, Mann-Whitney test and the receiver operating characteristics (ROC) calculations were performed with MedCalc (MedCalc Software). Results for continuous variables were expressed with the medians and standard deviations. Calculated $\mathrm{P}$ values of less than 0.05 were considered to indicate statistical significance. Correlation analyses were performed with Microsoft Excel 2002 SP-2 using the 'add trendline' functionality.

\section{Additional files}

Additional file 1: Figure S1: Amino acid sequence confirmation of the anchor peptide Ahx-ateeglkv (see Table 1). Print screen of the MS/MS spectra decoding of $\mathrm{m} / \mathrm{z} 515.795$ that was performed with PEAKS software (Bioinformatics Solutions). The unusual amino acid Ahx cannot be handled by the software and instead is displayed as Lysine $(L)$ that is an isomer of Ahx and thus produces a fragment with the same mass.

Additional file 2: Figure S2: Inhibition of protease-activity with iodoacetamide. The protease inhibitor iodoacetamide together with CP-RP and IS was added to a serum specimen from one tumor patient and incubated for $22 \mathrm{~h}$ prior to LC-MS analyis. lodoacetamide concentrations ranged from 5 to 25 and $100 \mathrm{mmol} / \mathrm{L}$. The CP-AP concentration of the serum specimen without iodoacetamide was set to $100 \%$. Measurements were performed in triplicate and the squares indicate median values, error bars indicate standard deviations. The exponential regression was calculated with Excel (Microsoft) and the coefficient of determination $\left(R^{2}\right)$ is shown in the graph.

Additional file 3: Figure S1: Inter day reproducibility of reporter peptide spiking. One serum specimen was measured three times on four different days. CP-AP mean value: $31.9 \mu \mathrm{mol} / \mathrm{L}$. SD: 3.3. CV: 10.2\%. The central box represents the values from the lower to upper quartile (25 to 75 percentile). The middle line represents the median. The horizontal line extends from the minimum to the maximum value.

\section{Abbreviations}

RP: Reporter Peptide; CRP: C-reactive protein; LC/MS: Liquid chromatography mass spectrometry; CV: Coefficient of variation; SD: Standard deviation; CP: Cancer procoagulant; MALDI-TOF-MS: Matrix-assisted laser desorption/ ionization-time of flight mass spectrometry; TP: Tumor patients; IC: Inflammatory controls; HC: Healthy controls; CP-RP: Reporter peptide; CP-AP: Anchor peptide; IS: Internal standard; HPLC: High pressure liquid chromatography; m/z: Mass to charge ratio; QC: Quality control; QCT: Pooled serum specimens from tumor patients designated as 'quality control tumor'; QCH: Pooled serum specimens from healthy controls designated as 'quality control healthy'; ROC: Receiver operating characteristic; AUC: Area under the curve; XIC: Extracted ion chromatogram; Ahx: Aminohexanoic acid; Abu:

2-aminobutyric acid; a.u.: Arbitrary units.

\section{Competing interests}

The authors declare that they have no competing interests.

\section{Authors' contributions}

PF planned the experiments and wrote the manuscript, VC and DY performed the mass spectrometric measurements and the data analyses. RH was responsible for the design of the study and MN participated in the manuscript preparation and revised it critically. All authors read and approved the final manuscript.

\section{Acknowledgement}

We gratefully acknowledge that the costs of publication were supported by the LESSER-LOEWE Foundation e.V.

\section{Author details}

'Institute for Clinical Chemistry, Medical Faculty Mannheim of the University of Heidelberg, University Hospital Mannheim, Theodor-Kutzer-Ufer 1-3, Mannheim 68167, Germany. ${ }^{2}$ III. Medical Clinic, Medical Faculty Mannheim of the University of Heidelberg, University Hospital Mannheim,

Theodor-Kutzer-Ufer 1-3, Mannheim D - 68167, Germany.

Received: 17 January 2012 Accepted: 4 April 2012

Published: 8 June 2012

\section{References}

1. Lopez-Otin C, Bond JS: Proteases: multifunctional enzymes in life and disease. J Biol Chem 2008, 283(45):30433-30437.

2. Ludwig T: Local proteolytic activity in tumor cell invasion and metastasis. Bioessays 2005, 27(11):1181-1191.

3. Gimeno-Garcia AZ, Santana-Rodriguez A, Jimenez A, Parra-Blanco A, NicolasPerez D, Paz-Cabrera C, Diaz-Gonzalez F, Medina C, Diaz-Flores L, Quintero E: Up-regulation of gelatinases in the colorectal adenoma-carcinoma sequence. Eur J Cancer 2006, 42(18):3246-3252.

4. Egeblad $M$, Werb Z: New functions for the matrix metalloproteinases in cancer progression. Nature reviews 2002, 2(3):161-174.

5. Gocheva V, Wang HW, Gadea BB, Shree T, Hunter KE, Garfall AL, Berman T, Joyce JA: IL-4 induces cathepsin protease activity in tumor-associated macrophages to promote cancer growth and invasion. Genes Dev 2010, 24(3):241-255.

6. Findeisen P, Peccerella T, Post S, Wenz F, Neumaier M: Spiking of serum specimens with exogenous reporter peptides for mass spectrometry based protease profiling as diagnostic tool. Rapid Commun Mass Spectrom 2008, 22(8):1223-1229.

7. Villanueva J, Nazarian A, Lawlor K, Tempst P: Monitoring peptidase activities in complex proteomes by MALDI-TOF mass spectrometry. Nat Protoc 2009, 4(8):1167-1183.

8. Peccerella T, Lukan N, Hofheinz R, Schadendorf D, Kostrezewa M, Neumaier $M$, Findeisen P: Endoprotease profiling with double-tagged peptide substrates: a new diagnostic approach in oncology. Clin Chem 2010, 56(2):272-280.

9. Dekker L, Burgers PC, Charif H, van Rijswijk AL, Titulaer MK, Jenster G, Bischoff R, Bangma CH, Luider TM: Differential expression of protease activity in serum samples of prostate carcinoma patients with metastases. Proteomics 2010, 10(12):2348-2358.

10. Somiari SB, Somiari Rl, Heckman CM, Olsen CH, Jordan RM, Russell SJ, Shriver CD: Circulating MMP2 and MMP9 in breast cancer - potential role in classification of patients into low risk, high risk, benign disease and breast cancer categories. Int J Cancer 2006, 119(6):1403-1411.

11. Findeisen P, Post S, Wenz F, Neumaier M: Addition of exogenous reporter peptides to serum samples before mass spectrometry-based protease 
profiling provides advantages over profiling of endogenous peptides. Clin Chem 2007, 53(10):1864-1866.

12. Witt I: Test systems with synthetic peptide substrates in haemostaseology. Eur J Clin Chem Clin Biochem 1991, 29(6):355-374.

13. Szaji E, Feher T, Medzihradszky KF: Investigating the quantitative nature of MALDI-TOF MS. Mol Cell Proteomics 2008, 7(12):2410-2418.

14. Yi J, Liu Z, Craft D, O'Mullan P, Ju G, Gelfand CA: Intrinsic peptidase activity causes a sequential multi-step reaction (SMSR) in digestion of human plasma peptides. J Proteome Res 2008, 7(12):5112-5118.

15. Rawlings ND, Morton FR, Kok CY, Kong J, Barrett AJ: MEROPS: the peptidase database. Nucleic Acids Res 2008, 36(Database issue):D320-D325.

16. Chechlinska M, Kowalewska M, Nowak R: Systemic inflammation as a confounding factor in cancer biomarker discovery and validation. Nature reviews 2010, 10(1):2-3.

17. Bland JM, Altman DG: Statistical methods for assessing agreement between two methods of clinical measurement. Lancet 1986, 1(8476):307-310.

18. Mielicki WP: Biochemistry of cancer procoagulant. Haemostasis 2001, 31(Suppl 1):8-10.

19. McDonald R: Quality assessment of quantitative analytical results in laboratory medicine by root mean square of measurement deviation. J Lab Med 2006, 30(3):111-117.

20. Findeisen $P$, Neumaier $M$ : Functional protease profiling for diagnosis of malignant disease. Proteomics Clin Appl 2012, 6(1-2):60-78.

21. Gordon SG, Benson B: Analysis of serum cancer procoagulant activity and its possible use as a tumor marker. Thromb Res 1989, 56(3):431-440.

22. Molnar S, Guglielmone H, Lavarda M, Rizzi ML, Jarchum G: Procoagulant factors in patients with cancer. Hematology (Amsterdam, Netherlands) 2007, 12(6):555-559.

23. Villanueva J, Nazarian A, Lawlor K, Yi SS, Robbins RJ, Tempst P: A sequencespecific exopeptidase activity test (SSEAT) for "functional" biomarker discovery. Mol Cell Proteomics 2008, 7(3):509-518.

24. van den Broek I, Sparidans RW, van Winden AW, Gast MC, van Dulken EJ, Schellens $J H$, Beijnen JH: The absolute quantification of eight inter-alphatrypsin inhibitor heavy chain 4 (ITIH4)-derived peptides in serum from breast cancer patients. Proteomics Clin Appl 2010, 4(12):931-939.

25. Murao N, Ishigai M, Yasuno H, Shimonaka Y, Aso Y: Simple and sensitive quantification of bioactive peptides in biological matrices using liquid chromatography/selected reaction monitoring mass spectrometry coupled with trichloroacetic acid clean-up. Rapid Commun Mass Spectrom 2007, 21(24):4033-4038.

26. Jeppsson JO, Kobold U, Barr J, Finke A, Hoelzel W, Hoshino T, Miedema K, Mosca A, Mauri P, Paroni R, et al: Approved IFCC reference method for the measurement of HbA1c in human blood. Clin Chem Lab Med 2002, 40(1):78-89.

27. Lin $\mathrm{S}$, Shaler $T A$, Becker $C H$ : Quantification of intermediate-abundance proteins in serum by multiple reaction monitoring mass spectrometry in a single-quadrupole ion trap. Anal Chem 2006, 78(16):5762-5767.

28. Bakun M, Karczmarski J, Poznanski J, Rubel T, Rozga M, Malinowska A, Sands D, Hennig E, Oledzki J, Ostrowski J, et al: An integrated LC-ESI-MS platform for quantitation of serum peptide ladders. Application for colon carcinoma study. Proteomics Clin Appl 2009, 3(8):932-946.

29. Diamandis E: Peptidomics for cancer diagnosis: present and future. J Proteome Res 2006, 5(9):2079-2082.

30. Falanga A, Gordon SG: Isolation and characterization of cancer procoagulant: a cysteine proteinase from malignant tissue. Biochemistry 1985, 24(20):5558-5567.

31. O'Mullan P, Craft D, Yi J, Gelfand CA: Thrombin induces broad spectrum proteolysis in human serum samples. Clin Chem Lab Med 2009, 47(6):685-693.

32. Niessen $\mathrm{S}$, Hoover $\mathrm{H}$, Gale AJ: Proteomic analysis of the coagulation reaction in plasma and whole blood using PROTOMAP. Proteomics 2011, 11(12):2377-2388.

33. Wildes D, Wells JA: Sampling the N-terminal proteome of human blood. Proc Natl Acad Sci U S A 2010, 107(10):4561-4566.

34. Murnane MJ, Shuja S, Del Re E, Cai J, lacobuzio-Donahue C, Klepeis V: Characterizing human colorectal carcinomas by proteolytic profile. In vivo (Athens, Greece) 1997, 11(3):209-216.

35. Gosalia DN, Denney WS, Salisbury CM, Ellman JA, Diamond SL: Functional phenotyping of human plasma using a 361-fluorogenic substrate biosensing microarray. Biotechnol Bioeng 2006, 94(6):1099-1110.
36. Watson DS, Jambunathan K, Askew DS, Kodukula K, Galande AK: Robust substrate profiling method reveals striking differences in specificities of serum and lung fluid proteases. Biotechniques 2011, 51(2):95-104.

doi:10.1186/1756-9966-31-56

Cite this article as: Findeisen et al:: Functional protease profiling with reporter peptides in serum specimens of colorectal cancer patients: demonstration of its routine diagnostic applicability. Journal of Experimental \& Clinical Cancer Research 2012 31:56.

\section{Submit your next manuscript to BioMed Central and take full advantage of:}

- Convenient online submission

- Thorough peer review

- No space constraints or color figure charges

- Immediate publication on acceptance

- Inclusion in PubMed, CAS, Scopus and Google Scholar

- Research which is freely available for redistribution

Submit your manuscript at www.biomedcentral.com/submit
C Biomed Central 